\title{
Size Determination by Phase-contrast Microscopy of the Reproductive Elements of Group A Streptococcal L-Forms
}

\author{
By C. P. A. VAN BOVEN*, HENRIETTE L. ENSERING \\ AND W. HIJMANS \\ Institute for Rheumatism Research, University Hospital, \\ Leiden, The Netherlands
}

(Accepted for publication I5 February 1968)

SUMMARY

Slide cultures consisting of small agar blocks were inoculated with the L-forms of two group A streptococci strains GL-8 and AED grown in broth. The size of the L-form elements was measured on photographs taken immediately after inoculation of the slide cultures. The diameter of the elements varied between 0.2 and $3.0 \mu$. The frequency distribution of the diameters has a bimodal form. This bimodality probably represents the size distribution of two classes of L-form elements which can arbitrarily be divided at the diameter of $0.7 \mu$.

The reproductive elements of the streptococcal L-forms were found, with a few exceptions, to have a minimal diameter of $0.7 \mu$ (average diameter $\mathrm{I} \cdot 4$ and $\mathrm{I} \cdot 2 \mu$, respectively; standard deviation $0.3 \mu$ ).

Slide culture experiments with concentrated filtrates of 0.45 filters showed that the reproductive elements present in the filtrates ranged in size from 0.8 to $\mathrm{I} \cdot 7 \mu$ with an average diameter of $\mathrm{I} \cdot 2 \mu$ (standard deviation $0.2 \mu$ ). The filterability of the streptococcal L-form might therefore be mainly due to the plasticity of the L-form elements.

\section{INTRODUCTION}

The occurrence of elements of widely differing sizes, with diameters ranging from the resolving power of the light microscope to several $\mu$, is characteristic for the L-forms of bacteria. With respect to the size of the elements capable of reproduction, the results of filtration experiments generally indicate that elements with a diameter of $0.35 \mu$ or even smaller are capable of reproduction (see van Boven, Ensering \& Hijmans, I968). However, studies of the reproductive L-form elements by phase-contrast microscopy (Weibull \& Lundin, 1962; Weibull, 1963; Taubeneck, 1962), with the micromanipulator (Roux, 1960) and in stained agar preparations (Dienes, 1967, 1968) have yielded values of $0.6-0.7 \mu$ or larger.

One of the factors considered to affect the passage of L-form elements through filter pores is their plasticity (van Boven et al. 1968). This may explain the differences between the results of filtration experiments and direct microscopic measurement; the size of the smallest reproductive L-form elements obtained by the filtration method tends to be too low.

* Present address: Laboratory of Microbiology, University of Utrecht, The Netherlands. 
The filtration experiments with the L-forms of two group A streptococci indicated that the size of the smallest reproductive elements varied between 0.45 and $0.65 \mu$ (van Boven et al. 1968). The present paper reports the results of microscopical size determinations of the L-form elements of the same two strains. The size distribution of the elements and the relation between size and reproductive capacity were determined by phase-contrast microscopy of slide cultures. To test the assumption that their plasticity allows L-form elements to pass membrane filters with pores smaller than these elements, the concentrated filtrates from $0.45 \mu$ Millipore membrane filters were studied by the same method. The minimal size of the L-form elements of the two strains of group A streptococci which commenced growth and developed into micro-colonies was $0.7 \mu$ or larger. The $\mathrm{L}$-form elements responsible for the development of growth in the $0.45 \mu$ filtrates had diameters of the same order of magnitude, i.e. $0.8 \mu$ or larger.

\section{METHODS}

Organisms and cultivation. The L-forms of the GL-8 and AED strains of group A streptococci and the methods of cultivation were described in the preceding paper (van Boven et al. 1968). For the slide cultures, the medium was supplemented with $10 \%(\mathrm{v} / \mathrm{v})$ inactivated horse serum.

Preparation of the slide cultures. Small agar blocks $(7 \times 7 \mathrm{~mm}$.) were cut out of I mm. thick agar plates and placed on conventional glass slides $(76 \times 26 \mathrm{~mm}$.). Inoculation was made either by streaking $0.2 \mathrm{ml}$. broth culture on the agar plate before cutting out the blocks or by placing a loopful of broth culture on the agar block. Coverslips $(22 \times 22 \mathrm{~mm}$.) were placed on the agar blocks, and the preparation was sealed off with a mixture of petroleum jelly (10\%) and paraffin. For counting and orientation in the slide cultures according to Taubeneck (I959), a Formvar film provided with a counting grid $(3 \times 3 \mathrm{~mm}$.; ruled in $60 \times 60$ squares of $50 \times 50 \mu)$ was attached to each coverslip before use. The slide cultures were incubated at $37^{\circ}$.

Phase-contrast microscopy. A Leitz Ortholux microscope (Ernst Leitz GMBH, Wetzlar, Germany) equipped with the Leitz phase-contrast system was used with the $\times 63$ dry objective (N.A. $0 \cdot 70$ ) or the $\times 90$ oil immersion objective (N.A. $1 \cdot 15$ ). Photographs were taken with either a micrographic camera or an automatic microscope camera (Leitz Orthomat Automatic Camera) with a green filter and Ilford Pan F film (Ilford Ltd, Ilford, Essex, England). Negatives were printed and enlarged to give a final magnification of 3600 ( $\times 90$ objective) or 2625 ( $\times 63$ objective).

Determination of particle size and reproductive capacity. The diameter of sharplyfocused single elements was measured on the photographic prints. For non-spherical elements the smallest diameter was taken. The measurements were done in $\mathrm{mm}$. to the nearest $0.1 \mathrm{~mm}$., and converted into $\mu$. To assess the accuracy of the method, the diameter of polysterene spheres with a known diameter of $0.81 \mu$ (Difco Bacto-Latex, 0.8I, Difco Laboratories, Detroit, Michigan, U.S.A.) were measured. The average diameter and standard deviation derived from measurements of 30 and 82 polysterene spheres with a $\times 63$ and $\times 90$ objectives, respectively, were $0.8 \mu \pm 0.09 \mu$ and $0.9 \mu \pm$ $0.04 \mu$.

The reproductive capacity of the elements was assessed from measurements done in photographs of the same fields made directly after inoculation and at regular intervals during incubation. Comparison of the diameters of the elements in the 
successive photographs showed which of the elements had developed into a microcolony.

Size of filterable elements. Standard Millipore filter membranes of type HA, pore size $0.45 \mu$, were employed. L-form suspensions were prepared and filtered as previously described (van Boven et al. 1968). The filtrates were concentrated by centrifugation at $50,000 \mathrm{~g}$ for $60 \mathrm{~min}$. in a Spinco Model $\mathrm{L}$ preparative ultracentrifuge equipped with a no. 30 rotor. Slide cultures were inoculated with the sediment.

\section{RESULTS}

\section{Microscopic appearance and particle-size distribution}

To study the microscopical aspect of the elements in L-form broth cultures of group A streptococci, slide cultures were prepared from cultures incubated for $15 \mathrm{hr}$ at $37^{\circ}$. Plate I, fig. I, 2 show the L-form elements of the GL-8 and AED strain on slide

\section{Table I. Size and relation between size and reproductive capacity of the L-form elements of the GL-8 and AED strain of group A streptococci}

Series I. Slide cultures were inoculated from I 5-hr L-form broth cultures and photographed immediately afterwards, using a phase contrast microscope with a $\times 90$ oil immersion objective. Particle diameters were measured on the enlarged photographic prints: final magnification $\times 3600$.

Series II. L-form broth cultures incubated for $9 \mathrm{hr}$ were used to inoculate the slide cultures. Photographs were taken immediately after inoculation and at regular intervals during incubation. A $\times 90$ oil immersion phase-contrast objective and a $\times 63$ dry phasecontrast objective were used in the experiments with the GL-8 and AED strains, respectively. The photographs were enlarged to a final magnification of $\times 3600$ and $\times 2625$ respectively.

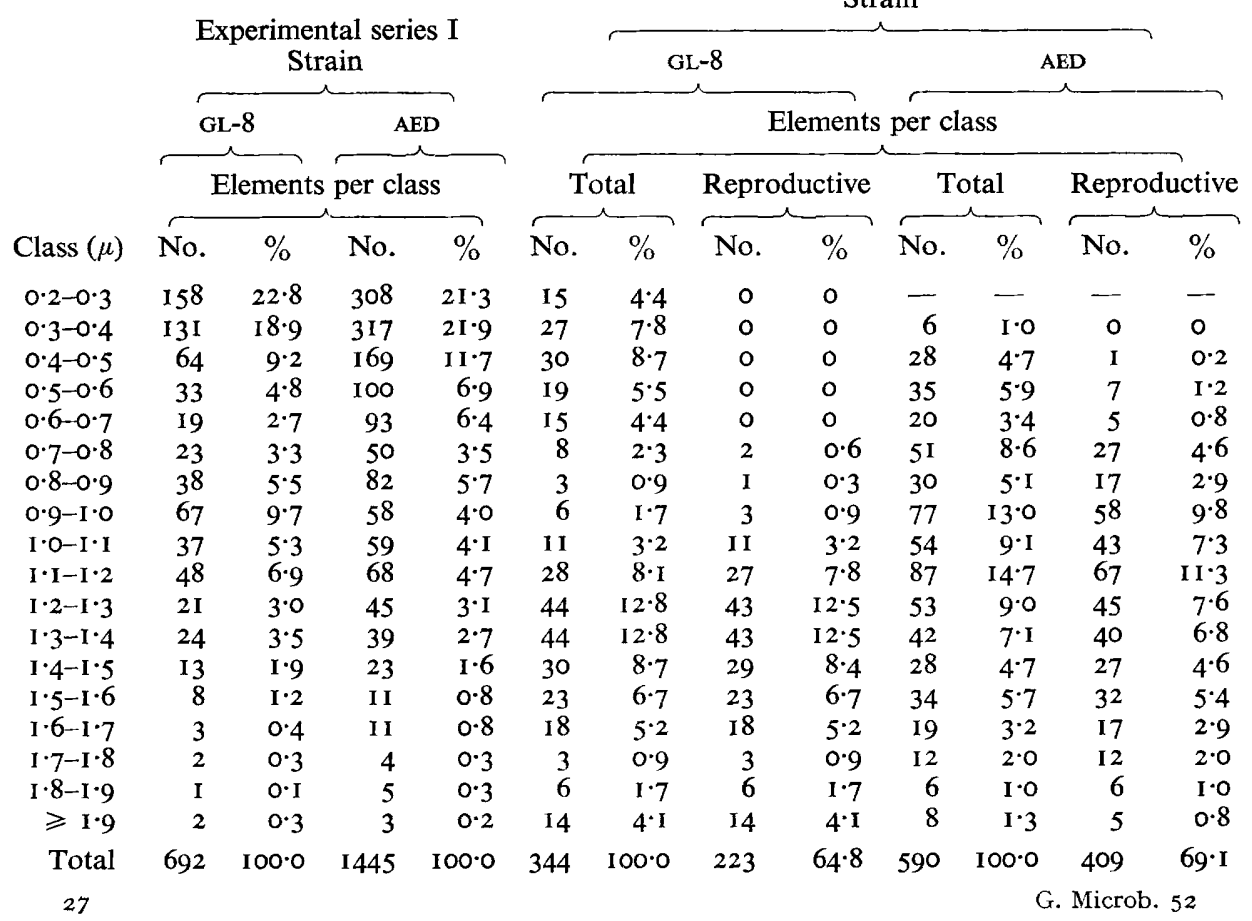

Experimental series II Strain 
cultures photographed immediately after inoculation. For comparison, Pl. 2, fig. 5 shows the appearance of a slide inoculated with sterile broth. The photographs demonstrate the pleiomorphic aspect of the L-form elements, especially with regard to size.
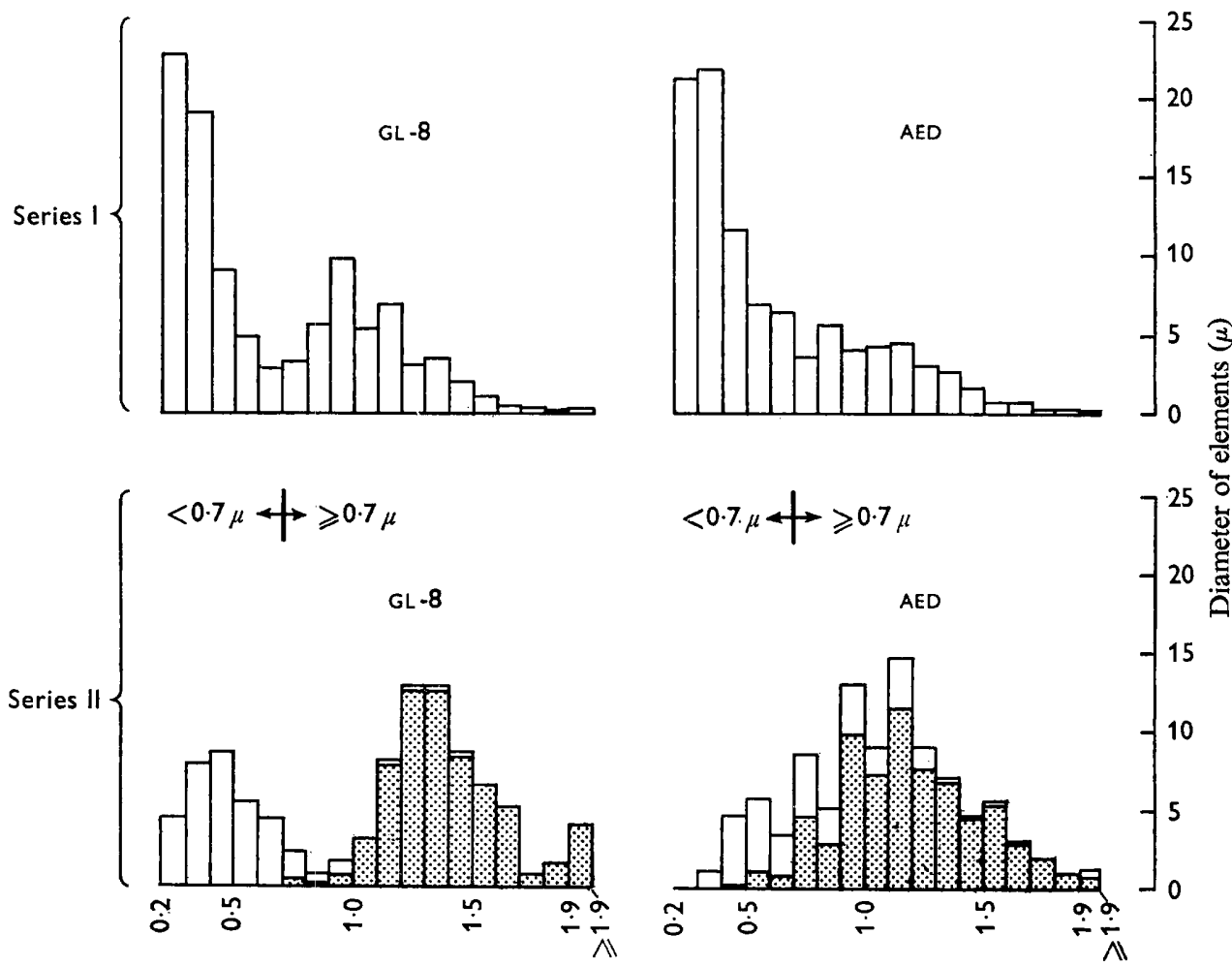

Frequency per cent in class range

Fig. I. Histograms of the diameters of L-form elements of the GL-8 and AED strains of group A streptococci. The histograms are based on the figures given in Table I.

Series I. Frequency distribution of element diameters in 15 -hr broth cultures.

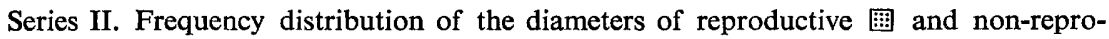
ductive $\square$ elements in 9-hr cultures.

Spherical and ovoid elements with diameters of about I $\mu$ and larger are clearly depicted, but the majority of the particles had diameters near the resolving power of the microscope. In the first series of experiments the diameters of the elements were measured on the enlarged photographic prints obtained from these slide cultures. Table I, series I shows the results of measurement of the diameters of 692 and 1445 L-form elements of the GL-8 and AED strains, respectively. In both cases more than half the elements had a diameter of less than $0.5 \mu$, about one-fourth fall between $0 \cdot 5-\mathrm{I} \cdot 0 \mu$, and the rest showed a diameter of $\mathrm{I} \cdot 0 \mu$ or larger. The largest elements observed had a diameter of $2 \cdot 5-3 \cdot 0 \mu$.

The frequency distribution of the diameters of the GL-8 L-form elements, as illustrated in Fig. I, series I, takes a bimodal form. This bimodality suggests that the 
L-form elements can be divided into two classes according to size. These classes were arbitrarily separated at the diameter of $0.7 \mu$. The bimodality is less clearly shown by the size distribution of the I445 AED L-form elements.

The average diameter and standard deviation of the two classes of L-form elements were identical for both the GL-8 and AED strain (i.e. $0 \cdot 4 \mu \pm 0 \cdot \mathrm{I} \mu$ and I I I $\mu \pm 0.3 \mu$ ). It should be noted that the average diameter and standard deviation of the smaller class are only approximate. The measurements obtained are incomplete because some of the elements of this class probably had diameters below the resolving power of the microscope.

\section{Reproductive capacity of elements of different sizes}

The reproductive capacity of the $L$-form elements, i.e. the capacity to develop into a micro-colony, was assessed in the second series of experiments. Growth was determined by comparing of elements in photographs of the same fields taken immediately after inoculation and at regular intervals during incubation. This is illustrated in P1. 2, fig. 6, 7, 8. Since L-form cultures of the GL-8 and AED strains yielded a maximal number of viable elements after $9 \mathrm{hr}$ of incubation, in these experiments the slide cultures were inoculated with broth cultures incubated for that period.

Table I, series 2 shows the results; the total number per class and the number of reproductive elements present are given. The size distribution of the GL- 8 L-form elements, as demonstrated by the histogram in Fig. I series 2, again indicated the existence of a class of L-form elements with diameters of $0.7 \mu$ or larger, and a class of elements with diameters less than $0 \cdot 7 \mu$. The observations for the AED strain were made with a $\times 63$ objective; the resolving power of this system is about $0.5 \mu$. A small proportion of the class of elements with diameters less than $0.7 \mu$ could therefore be measured. The few measurements obtained below the value $0.5 \mu$ are only approximate.

The elements which developed into a micro-colony had, with a few exceptions in the AED strain, a diameter of $0.7 \mu$ or more. The size distribution of the growing elements (as shown by Table I, series 2 and by the histograms of Fig. I, series 2) coincides with the size distribution of the L-form elements belonging to the class of elements with diameters of $0 \cdot 7 \mu$ or more. The parameters of these size distributions, the mean diameter, and the standard deviation, were the same, i.e. $1 \cdot 4 \mu \pm 0 \cdot 3 \mu$ in the GL- 8 strain and $\mathrm{I} \cdot 2 \mu \pm 0 \cdot 3 \mu$ in the AED strain.

\section{Size of reproductive elements in $0.45 \mu$ filtrates}

Plate I, fig. 3, shows a slide culture inoculated with the concentrated filtrate from a $0.45 \mu$ Millipore membrane filter. A large number of particles with diameters near the resolving power of the microscope and a few larger elements are visible. Observation of these slide cultures during incubation showed that only the large elements developed into micro-colonies, as can be seen by comparison of Pl. I, fig. 3, 4. No growth was observed of any of the very numerous smaller elements. The data on the large elements are limited because, notwithstanding the concentration, the number of these elements was low and they were therefore difficult to find under the microscope. A total of 49 elements which had developed into micro-colonies were observed and photographed. Measurements of the diameter of these elements on the photographs taken immediately after inoculation showed the size of the elements to vary between 0.8 and 
$\mathrm{I} \cdot 7 \mu$. The average diameter and standard deviation of the growing elements, i.e. $\mathrm{I} \cdot 2 \mu \pm 0.2 \mu$, are similar to the values found for the class of reproductive elements.

\section{DISCUSSION}

The data in the present paper show a relationship between size as measured microscopically and reproductive capacity of the L-form elements of group A streptococci. A minimal size of $0.7 \mu$ appears to be essential for reproduction, since only elements with a diameter of $0.7 \mu$ or larger developed into micro-colonies. Microscopical studies of the L-form of group A streptococci (Dienes, 1967, I968) and other bacteria (Roux, 1960; Taubeneck, 1962; Weibull \& Lundin, 1962; Weibull, 1963; Fodor \& Miltényi, 1964; Dienes, I968) have also indicated that after transfer to fresh agar medium, growth developed exclusively from elements with a diameter of $0.6-0.7 \mu$ or larger. The pleomorphic character of the L-form is revealed by the variation in size of the viable elements and also by the occurrence of elements with considerably smaller diameters.

The diameters of the streptococcal L-form elements were measured on photographs obtained by phase-contrast microscopy. With phase-contrast microscopy, the resulting modified diffraction gradients usually do not represent the actual boundaries of the objects (Ross, 1957; Ross \& Galavazi, I964). In the present study the 'visible size' was measured according to Chairman (1963) as the distance between the outer visible limits of the diffraction gradients. To assess the error inherent in this procedure, measurements were made on polysterene spheres of known diameter. The results indicate that, at least with particles of this diameter, a reliable estimation of the size of objects can be obtained.

In a previous study (van Boven et al. 1968) it has been shown that reproductive elements of the L-form of the same group A streptococcal strains passed through membrane filters with $0.45 \mu$ porosities. The results suggested that passage through this filter might be due to the plasticity of the L-form elements. The demonstration in the present study of L-form elements with diameters between 0.8 and $1 \cdot 7 \mu$ in the concentrated $0.45 \mu$ filtrates, provides supporting evidence for this assumption. Only these elements were found to develop into micro-colonies; the numerous smaller elements present in the filtrates showed no sign of multiplication during the period of observation. The filterability of the streptococcal L-form might therefore be mainly due to the plasticity of the L-form elements, which would explain the discrepancy between the results of the filtration method and the microscopic measurements with respect to the size of the reproductive L-form elements.

The role of the elements with diameters less than $0.6-0.7 \mu$ is not yet clear. These elements, which range down to $0.05 \mu$ as measured electron microscopically (Thorsson \& Weibull, 1958 $a, b$; Weibull, Mohri \& Afzelius, 1965; Ryter \& Landman, I964; Coussons \& Cole, I968), are produced during growth of the bacterial L-form. Dienes (Dienes, I967, 1968; Dienes \& Madoff, 1966, I968; Dienes \& Bullivant, 1967) observed multiplication of these elements when embedded into the agar gel or in the internal structure of membrane filters. In a liquid medium or after transfer to fresh agar medium, multiplication of these elements did not occur; under these circumstances growth developed from larger elements, the so-called 'large bodies'. The latter observation is in accordance with the results of the present study. Confirmation of the former observation cannot be provided by our experiments. The same holds for 
the hypothesis put forward by Klieneberger-Nobel (1962) that the small granular L-form elements can develop only when embedded in a matrix of delicate slime. Separation of the granules from the matrix would result in loss of reproductive capacity. The assumption that multiplication of small L-form elements is possible only in the coherence of the L-form colony would explain the failure to demonstrate development of these elements in the present slide-culture experiments. It would also explain the penetration of the streptococcal L-form through membrane filters with low porosities (van Boven et al. 1968), but not the filterability of the L-form elements.

Further evidence which seems to exclude the possibility that the 'small' L-form elements play a significant role in the reproduction of the bacterial L-form has been provided by biochemical studies (Weibull \& Beckman, I96r; Sensenbrenner, BaderHirsch, Terranova \& Mandel, I964) indicating a low DNA content of the small L-phase elements, and by electron-microscopical studies (Ryter \& Landman, 1964; Coussons \& Cole, I968) showing the absence of a nuclear region in a considerable number of the small elements.

The bimodality of the size distribution of the group A streptococcal L-form elements, furthermore, suggests the existence of two definite populations. A similar size distribution has been found for the L-form elements of a Proteus L-form (Weibull \& Lundin, 1962). The majority of the elements in the size range of $0.6-0.7 \mu$ are apparently capable of growing and increasing in size, which explains the bimodal size distribution.

In conclusion, the results of the previously reported filtration experiments (van Boven et al. 1968) and of the present microscopical study indicate a size between 0.6 and $0.7 \mu$ for the minimal reproductive elements of the L-forms of two group A streptococcal strains. Although reproduction of elements of smaller sizes under certain conditions can not be excluded, reproduction of these elements did not occur under the experimental conditions. To elucidate the role of these elements and to further our understanding of the reproductive physiology of the bacterial L-form, detailed analysis of the small elements, especially with respect to the distribution of DNA in the individual elements, will be required.

\section{REFERENCES}

van Boven, C. P. A., Ensering, H. E. \& Hijmans, W. (1968). Size determination by the filtration method of the reproductive element of group A streptococcal L-forms J. gen. Microbiol. 52, 403.

Chatrman, W. N. (1963). Some experiments concerning the limitations and errors in size measurement of small objects by visual microscopy. $J l R$. microsc. Soc. 82, 81.

Coussons, R. T. \& Cole, R. M. (1968). The size and replicative capacities of small bodies of group A streptococcal L-forms. In The Present Status of Research on Group A Streptococcus. Ed. by R. Caravano. Amsterdam: Excerpta Medica Foundation.

DIENES, L. (1967). Morphology and reproductive processes of the L-forms of bacteria. I. Streptococci and staphylococci. J. Bact. 93, 693.

DiEnEs, L. (1968). Morphology and reproductive processes of bacteria with defective cell wall. In Microbial Protoplasts, Spheroplasts and L-forms. Ed. by L. B. Guze. Baltimore: The Williams and Wilkins Co.

Dienes, L. \& Bullivant, S. (1967). Comparison of the morphology of PPLO and L-forms of bacteria with light and electron microscopy. Ann. N.Y. Acad. Sci. 143, 7 I 9.

Dienes, L. \& MADOFF, S. (I966). Development and growth of L-forms of bacteria and PPLO on membrane filters. Proc. Soc. exp. Biol. Med. I21, 334. 
DiEnEs, L. \& MADOFF, S. (I968). Comparison of the morphology of PPLO and L-forms of bacteria with light and electron microscopy. In The Present Status of Research on Group A Streptococcus. Ed. by R. Caravano. Amsterdam: Excerpta Medica Foundation.

FODOR, M. \& MILTÉNYI, L. (1964). Studies on L-forms of Staphylococcus aureus strains of different antibiotic and phage sensitivity. Acta microbiol. hung. Ir, 155.

KLIENEBERGER-NoBel, E. (I962). Pleuropneumonia-like Organisms (PPLO) Mycoplasmataceae. London and New York: Academic Press.

Ross, K. F. A. (I957). The size of living bacteria. Q. Jl microsc. Sci. 98, 435.

Ross, K. F. A. \& GALAVAZI, G. (1964). The size of bacteria, as measured by interference microscopy. Jl R. microsc. Soc. 84, 13.

Roux, J. (1960). La multiplication des formes L. Annls Inst. Pasteur, Paris 99, 286.

RYTER, A. \& LANDMAN, O. E. (1964). Electron microscope study of the relationship between mesosome loss and the stable L-state (or protoplast state) in Bacillus subtilis. J. Bact. 88, 457.

Sensenbrenner, M., Bader-Hirsch, A. M., Terranova, T. \& Mandel, P. (1964). Recherches sur le bacille Proteus $P$ I 8 et les formes $L$ qui en dérivent. I. Étude quantitative des acides ribo- et désoxyribonucléiques. Bull. Soc. Chim. biol. 46, 483.

TAUBENECK, U. (1959). New Grid-replica for precise localization in slide cultures. J. Bact. 77, 506.

TAUBENECK, U. (I962). Untersuchungen über die L-Form von Proteus mirabilis Hauser. II. Entwicklung und Wesen der L-Form. Z. allg. Mikrobiol. 2, I32.

Thorsson, R. C. \& Weibull, C. (1958a). Electron microscopy of a stable Proteus L-form. Nature, Lond. I8r, 1348.

Thorsson, R. C. \& Weibull, C. (1958b). Studies on the structure of bacterial L-forms, protoplasts and protoplast-like bodies. J. Ultrastruct. Res. x, $4 \mathrm{I} 2$.

Weibull, C. (1963). Size of minimal reproductive units of bacterial L-forms. Proc. Soc. exp. Biol. Med. $\mathrm{Ir}_{3}, 32$.

Weibull, C. \& BeCKman, H. (I961). Chemical and metabolic properties of various elements found in cultures of a stable L-form. J. gen. Microbiol. 24, 379.

WeIBull, C. \& Lundin, B-M. (1962). Growth of elements of various sizes found in cultures of a stable Proteus L-form. J. gen. Microbiol. 27, 241.

Werbull, C., Mohri, T. \& Afzelius, B. A. (1965). The morphology and fine structure of small particles present in cultures of a Proteus L-form. J. Ultrastruct. Res. 12, 8I.

\section{EXPLANATION OF PLATES}

Photographs of slide cultures of L-forms of Group A Streptococcus prepared according to Taubeneck (1959). The magnification is $\times 1260$.

\section{Plate I}

Fig. I. L-form elements of the GL-8 strain of Group A Streptococcus. The slide culture was photographed immediately after inoculation with a $15 \mathrm{hr}$ broth culture.

Fig. 2. L-form elements of the AED strain. Same as Fig. I.

Fig. 3. Slide culture of GL-8 L-form elements immediately after inoculation. The culture had been filtered through a $0.45 \mu$ filter.

Fig. 4. The same field as in Fig. 3 after 24 -hr incubation. The micro-colonies have developed from the two elements with diameters of 0.8 and $1 \cdot 7 \mu$.

\section{Plate 2}

Fig. 5. Photograph of control slide culture inoculated with sterile broth.

Fig. 6. Slide culture immediately after inoculation with a $9 \mathrm{hr}$ GL-8 L-form culture.

Fig. 7. The same field as in Fig. 6 after $\mathrm{I}$ hr incubation.

Fig. 8. The same field as in Fig. 6 and 7. Note that the micro-colonies have developed from the large elements after $3 \mathrm{hr}$ incubation. 


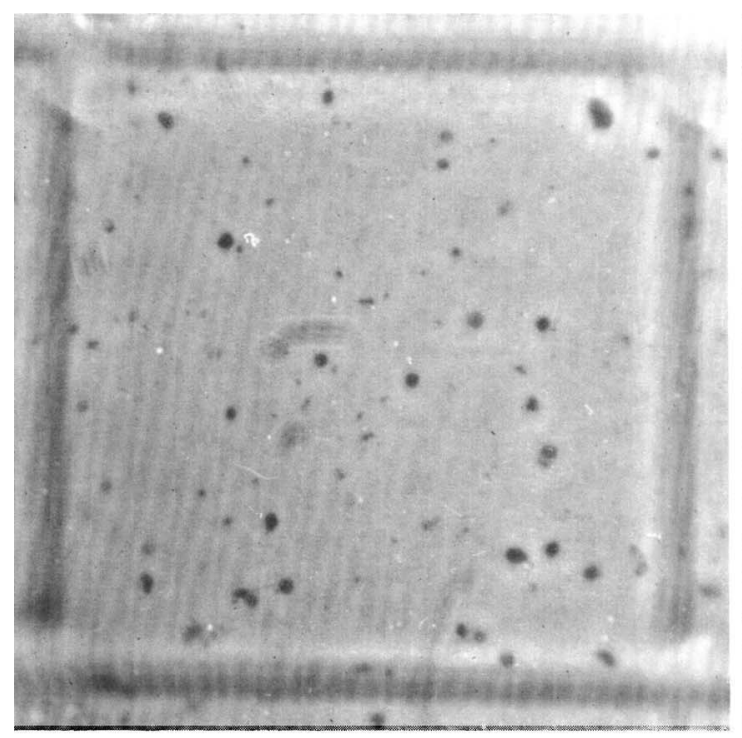

Fig. I

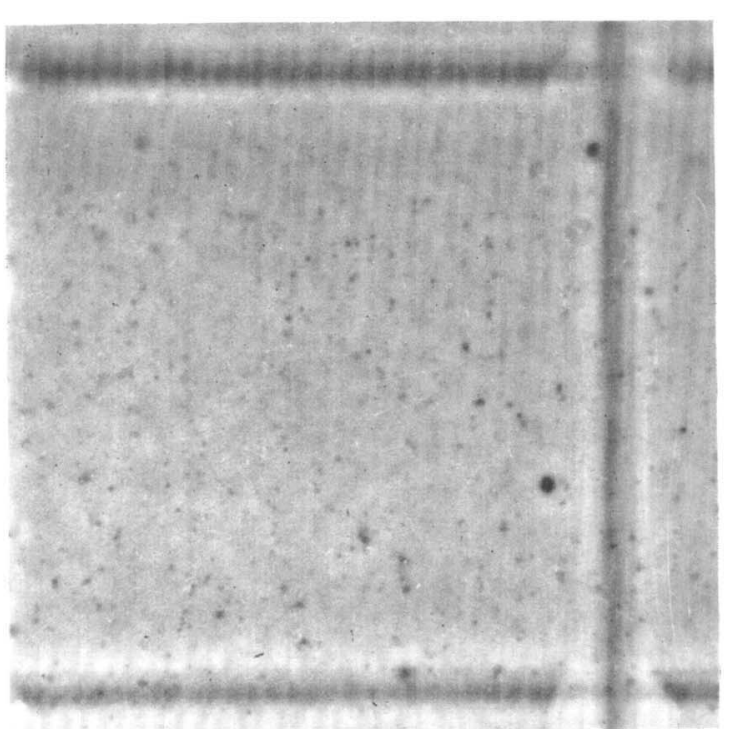

Fig. 3

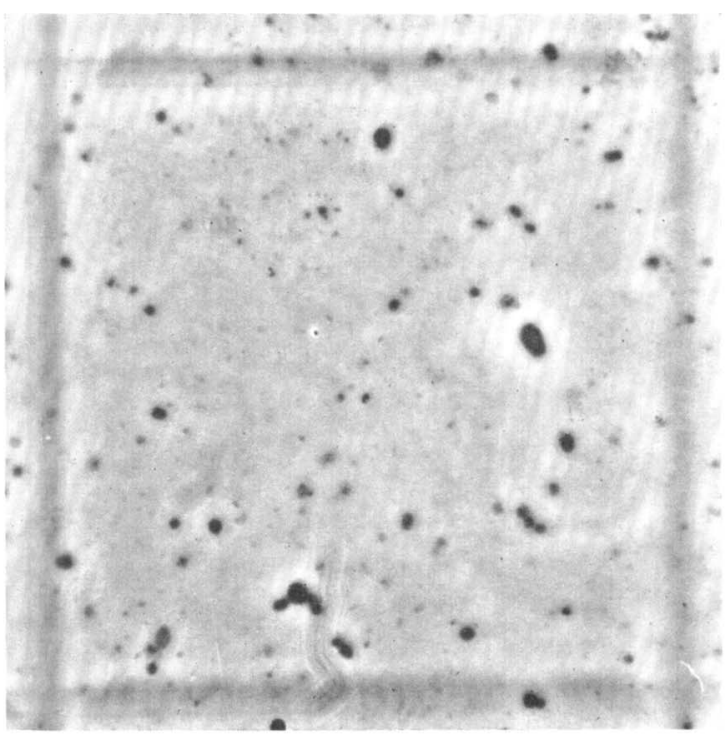

Fig. 2

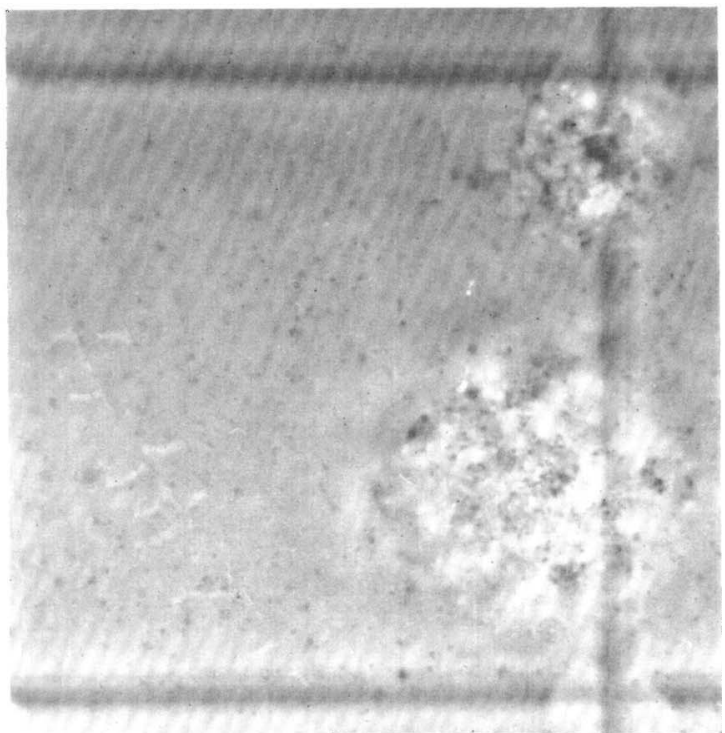

Fig. 4 
Journal of General Microbiology, Vol. 52, No. 3

Plate 2

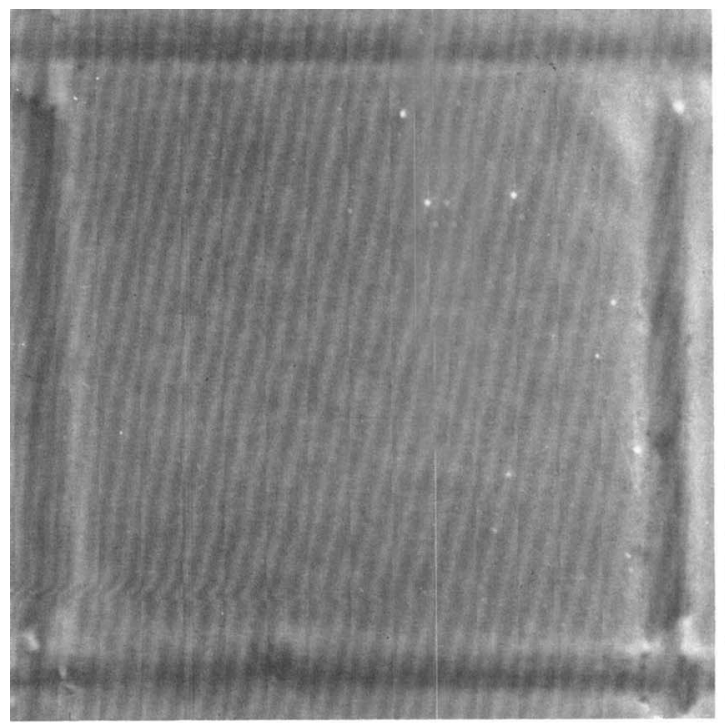

Fig. 5

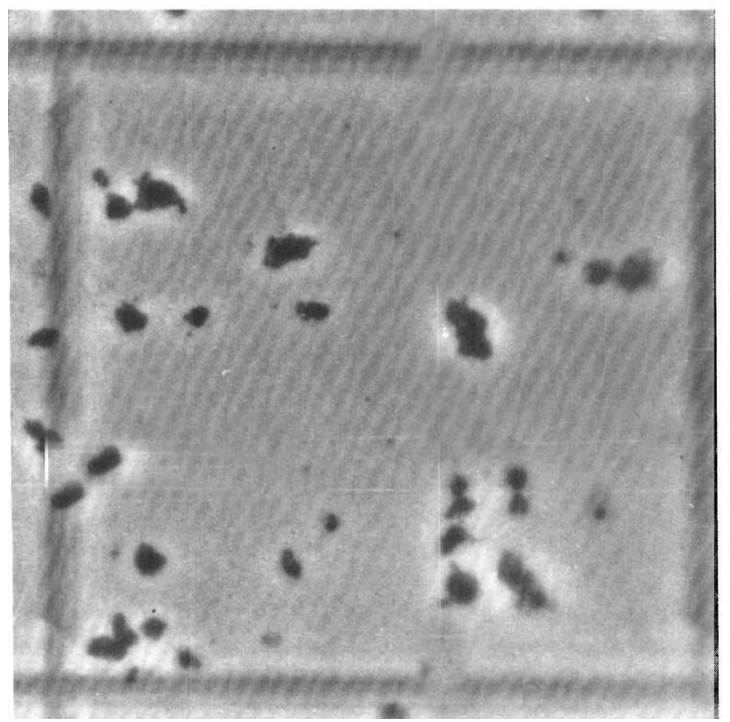

Fig. 7

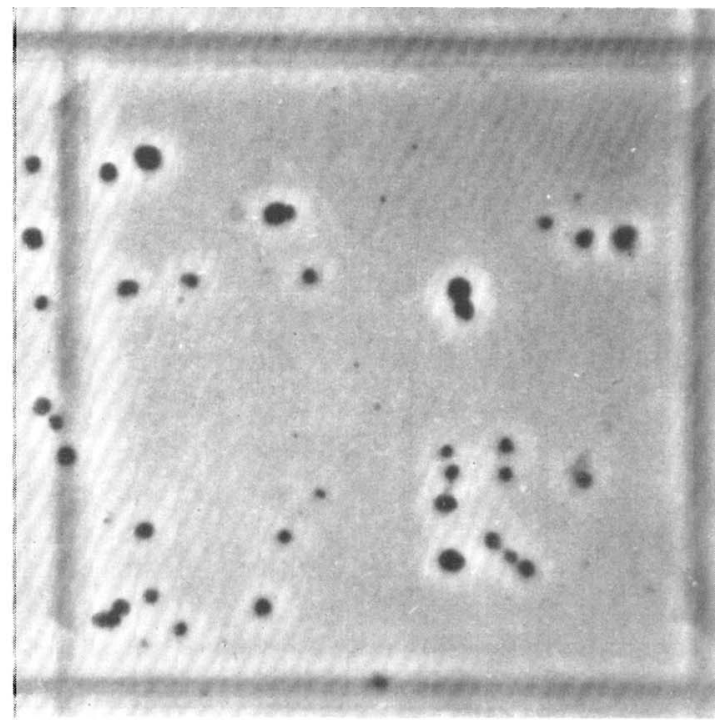

Fig. 6

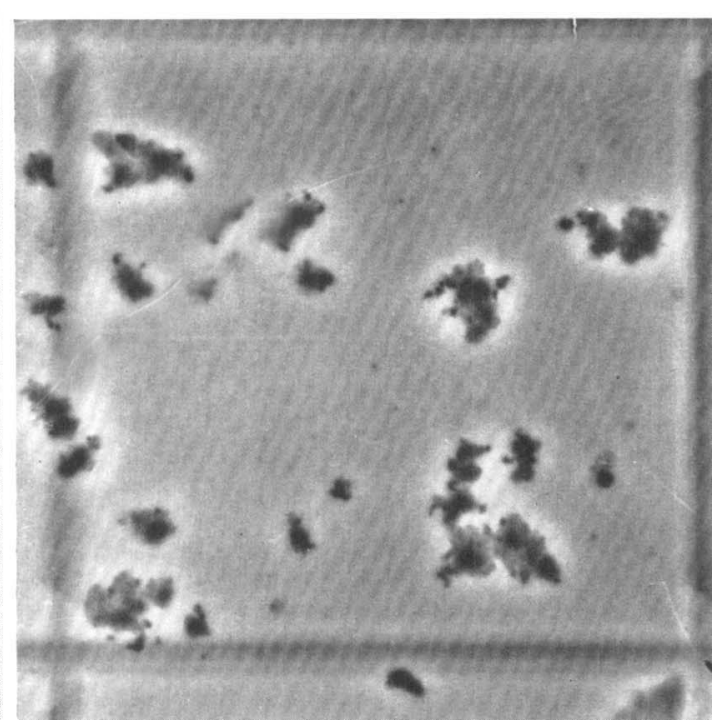

Fig. 8

C. P. A. van BOVEN, H. L. ENSERING aNd W. HIJMANS 